\title{
EFECTO DE LA CARGA DE SULFITO DE SODIO EN EL PULPAJE NSSC DE E. GLOBULUS
}

\section{EFFECT OF SODIUM SULFITE CHARGE IN NSSC PULPING OF E. GLOBULUS}

\author{
Miguel Angel Pereira ${ }^{1}$, Rudolf Patt ${ }^{2}$, Othar Kordsachia ${ }^{3}$
}

\section{RESUMEN}

El presente trabajo tuvo como principal objetivo determinar el efecto de la adición de una alta carga de sulfito de sodio $\left(\mathrm{Na}_{2} \mathrm{SO}_{3}\right)$ para la sustitución de otras fuentes alcalinas $\left(\mathrm{Na}_{2} \mathrm{CO}_{3}\right.$ o $\left.\mathrm{NaOH}\right)$ sobre el pulpaje de alto rendimiento al sulfito neutro. En la etapa química se utilizó sólo sulfito de sodio $\left(\mathrm{Na}_{2} \mathrm{SO}_{3}\right)$ como agente deslignificante y en la etapa mecánica un refinador Bauer de 12". Se obtuvieron pulpas con rendimientos variables entre 70 y $90 \%$ y se verificó que las propiedades físico-mecánicas de las pulpas aumentan considerablemente a medida que el rendimiento baja. Al mismo tiempo disminuyeron, notablemente, el consumo energético de desfibración y el de refinación. Pulpas con rendimientos cercanos al $80 \%$, fabricada con una alta dosis de sulfito de sodio presentaron propiedades físico-mecánicas similares a una pulpa química. La mayor carga de sulfito no significó un aumento considerable del consumo de reactivos, especialmente a altos rendimientos. El buen desarrollo de propiedades físicomecánicas se debe, probablemente, a la alta proporción de hemicelulosas que presenta la pulpa y a la selectividad del proceso para remover lignina desde la lámina media, lo que favorece la obtención de fibras íntegras y con un menor esfuerzo mecánico.

Palabras Claves: Pulpaje semiquimico (NSSC), Eucalyptus globulus, alto rendimiento.

\begin{abstract}
This paper presents experimental results on the effect of adding high doses of sodium sulphite $\left(\mathrm{Na}_{2} \mathrm{SO}_{3}\right)$ to replace other sources of alkali, such as $\mathrm{Na}_{2} \mathrm{CO}_{3}$ or $\mathrm{NaOH}$, in high-yield Neutral Semichemical Pulping (NSSC) process. Sodium sulfite $\left(\mathrm{Na}_{2} \mathrm{SO}_{3}\right)$ was used as delignification agent in the chemical stage of eucalyptus globulus pulping. A 12 " Bauer refiner was employed in the mechanical stage. Pulp yields ranged within $70-90 \%$ and, results show that physical and mechanical properties increased significantly as yields decreased. Moreover, the defibration energy consumption and beatings energy also showed a significant decrease.

Those pulps featuring yields near $80 \%$, obtained with high sodium sulfite loads, presented similar physical and mechanical properties as chemical pulps. At the largest sulfide dose used here, no significant increase in chemical consumption was observed, especially at high yields. This study shows that physical and chemical properties are enhanced by high hemicelluloses content, and process selectivity to remove lignin from the middle lamella, which leads to a fiber integrity and reduced mechanical stress.
\end{abstract}

Keyword: Semichemical pulp (NSSC), Eucalyptus globulus, high yield pulp.

\footnotetext{
El trabajo fue realizado en el Instituto de química y tecnología química de la madera de la Universidad de Hamburgo, Alemania.

${ }^{1}$ Laboratorio de Productos Forestales, Departamento de ingeniería Química, Universidad de Concepción, Casilla 160C, correo 3 Concepción.

${ }^{2}$ Institute for Wood Chemistry, department of biology, University of Hamburg, Germany.

${ }^{3}$ Federal Research Institute. Department for Wood Technology, Hamburg Germany.

Recibido: 16.09.2010. Aceptado: 26.04.2011

Autor para correspondencia: miguelpereira@udec.cl
} 


\section{INTRODUCCIÓN}

La diversificación del uso de pulpas semiquímicas de alta calidad en la fabricación de diversos tipos de papeles; para embalaje y empaque, papeles absorbentes e higiénicos y, principalmente en papeles para impresión y escritura, sumado a la flexibilidad para operar a pequeñas escalas ha devuelto el interés de utilizar estos procesos para la obtención de pulpa papelera (Area et al. 2001, Xu 2009).

La utilización de maderas latifoliadas (como los eucaliptos) en procesos semiquímicos de alto rendimiento, debido fundamentalmente a su bajo contenido de lignina, ha sido una de las variante más estudiadas durante el último tiempo (Xu 2009). Sin embargo, el uso de maderas duras presenta algunas desventajas, como por ejemplo; el alto deterioro de las fibras durante la etapa mecánica del proceso. Lo anterior obliga a optimizar la etapa química del proceso, de manera de promover una etapa mecánica menos intensiva, que permita la obtención de fibras más integras y con un menor consumo energético (Ingruber 1985, Area et al. 2004, Xu 2009).

La dificultad de recuperar los licores al sulfito (Ingruber 1985) ha llevado a una constante optimización el uso de reactivos en la etapa química del proceso, principalmente para disminuir costos (Area et al. 2002). Por la misma situación existe una búsqueda constante de usos alternativos a la tradicional recuperación de lignosulfonatos de los licores de cocción (Area et al. 2000a,b; Monsalve 2010). Soluciones alternativas pasan por integrar el sistema de recuperación de reactivos con una planta kraft, situación que en Chile pudiera ser ventajosa debido al gran desarrollo de esta industria, la posibilidad de reducir los make up de reactivos y el interés de elevar la capacidad generadora de electricidad. Por otra parte, el continuo desarrollo de la biorefinería forestal, que favorece la búsqueda de nuevos usos para materiales lignocelulósicos y el desarrollo de procesos energéticamente más eficientes de recuperación de reactivos pueden abrir una ventana más amplia de desarrollo para los procesos al sulfito, especialmente de alto rendimiento (Périn-Levasseur et al. 2010, Kordsachia 2002).

Tradicionalmente, el pulpaje semiquímico al sulfito neutro (NSSC), ocupa entre sus reactivos una fuente alcalina $\left(\mathrm{Na}_{2} \mathrm{CO}_{3} \mathrm{o} \mathrm{NaOH}\right)$ que actúa como tampón para procurar que el $\mathrm{pH}$ final de la solución de cocción no descienda a valores inferiores a 7.0. Dado el alto contenido de grupos acetilos en las xilanas de maderas latifoliadas, el control del pH, en este caso, resulta particularmente importante (Evtuguin y Neto 2007, Zanuttini et al. 1998, Zanuttini et al. 2005).

Area et al. (2002), determinaron que la utilización de $\mathrm{Ca}_{2} \mathrm{CO}_{3}$ durante el pulpado NSSC de eucaliptos tiene un pequeño efecto positivo sobre las propiedades físico-mecánicas de la pulpa y un efecto negativo sobre la blancura final de la misma. Al contrario, concluyen en su trabajo que el aumento de la fracción de $\mathrm{Na}_{2} \mathrm{SO}_{3}$ en la carga de reactivos, posee un efecto positivo sobre ambas propiedades. $\mathrm{El} \mathrm{Na}_{2} \mathrm{SO}_{3}$ es un álcali débil que en solución presenta un $\mathrm{pH}$ de $9,7 \mathrm{y}$, dado sus características, puede ser usado como agente regulador de $\mathrm{pH}$.

Este trabajo se centró en la premisa de que tanto el proceso de pulpaje al sulfito neutro, como las propiedades de la pulpa resultante, pueden ser mejorados al reemplazar la fuente alcalina tradicional del pulpaje NSSC $\left(\mathrm{Ca}_{2} \mathrm{CO}_{3}\right.$ o NaOH) por una mayor dosificación de $\mathrm{Na}_{2} \mathrm{SO}_{3}$. Por otra parte, al operar en condiciones más neutrales y/o ligeramente ácidas, las reacciones de sulfonación deberían verse favorecidas (Rydholm 1965).

En una primera etapa se estableció una relación entre la carga de reactivos y el desempeño pulpable en términos de rendimiento, consumo de reactivos y grado de sulfonación de la pulpa y, en una segunda, se estudio el efecto del rendimiento pulpable y la carga de reactivos sobre las propiedades físico-mecánicas de la pulpa y su blanqueabilidad. Como materia prima se eligió E. globulus, por su bajo contenido de lignina y su amplia disponibilidad en Chile. 


\section{MATERIALES Y MÉTODOS}

La etapa química del proceso se realizó en un digestor de laboratorio del tipo MK-System de 7L. Las cocciones se realizaron con $1 \mathrm{~kg}$ de madera base seca, razón licor/madera (L/M) igual a 4, temperatura máxima de cocción de $170^{\circ} \mathrm{C}$ y tiempo de calentamiento de $90 \mathrm{~min}$. La carga de $\mathrm{Na}_{2} \mathrm{SO}_{3}$ varió entre $5.9 \%$ y $35.4 \%$. Antes de la cocción las astillas fueron tratadas con vapor a 1 bar durante 30 minutos.

La etapa mecánica del proceso se realizó en un refinador de discos Sprout-Bauer de 12" diámetro. La desfibración se realizó haciendo pasar 3 veces el material a través del refinador de manera secuencial, con abertura entre platos de 2.0, 0.75 y $0.25 \mathrm{~mm}$ respectivamente. La consistencia de desfibración varió desde $4 \%$ en la primera pasada hasta $2 \%$ en la última.

El pulpaje fue evaluado mediante su rendimiento total determinando la humedad a peso constante en estufa a $105^{\circ} \mathrm{C} \pm 1^{\circ} \mathrm{C}$ de la pulpa final, de manera que el rendimiento corresponde al peso seco de la pulpa final obtenida dividida por el peso inicial seco de la madera. El grado de sulfonación de la pulpa fue determinado de acuerdo a la norma SCAN-CM 65:02.

La pulpa fue blanqueada en bolsas de polietileno utilizando $250 \mathrm{~g}$ (bps) en una secuencia QPFAS, previa optimización de las condiciones aquí utilizadas. La etapa de quelación $(\mathrm{Q})$ se realizó con una carga de $0,1 \%$ de DTPA a $60^{\circ} \mathrm{C}$ y 60 minutos. La etapa de peróxido (P), se llevó a cabo variando la carga de peróxido $(1-5 \%)$, tiempo $(120$ y $180 \mathrm{~min})$ y temperatura $\left(70-80^{\circ} \mathrm{C}\right)$ de reacción. La etapa final reductora se realizó con una carga de $0,5 \%$ de ácido formamidinsulfínico (FAS) a $98^{\circ} \mathrm{C}$ y $10 \%$ de consistencia y $\mathrm{pH}$ inicial 10,5.

La blancura final de la pulpa blanqueada fue determinada de acuerdo a norma SCAN C-11:75.

La pulpa blanqueada fue sometida a refinación mecánica en un molino Jokro de acuerdo a ISO 5264/ III-1979, y las hojas para los ensayos mecánicos de acuerdo a ISO 5269/2-1980 en una formadora de hoja de la marca Frank (Rapid-Köthen). El grado de refino (drenabilidad) se determinó de acuerdo a norma ISO 5267/1-1979, el Gramaje fue determinado de acuerdo a norma Tappi T 410 om-93, Tensión, explosión y rasgado Elmendorf de acuerdo a Tappi T 404 om-92, Tappi T 403 om-91 y Tappi T 414 om-88. Los cálculos de índices de tensión, rasgado y explosión de acuerdo a TAPPI 220 sp-96.

Debido a la alta carga de madera empleada y su disponibilidad, la estimación del error relativo de los resultados presentados en este trabajo se determinó mediante un análisis de reproducibilidad del pulpaje. Se escogió una condición de pulpaje y metodología de desfibrado $\left(170^{\circ} \mathrm{C}, 16,5 \% \mathrm{Na}_{2} \mathrm{SO}_{3}\right.$ y 60 min de cocción) y se repitió el ensayo 4 veces. El error asociado a estos cuatro ensayos se determinó como la desviación estándar respecto a la media. Se estimó que los ensayos a otras condiciones tendrían la misma variabilidad. Para el caso del rendimiento el error resultó $\pm 0,92 \%$, por tanto diferencias por sobre $2 \%$ pueden ser consideradas significativas. Adicionalmente para cada pulpaje se estimó la propagación del error debido al cálculo mediante el análisis de derivadas parciales, verificando que se mantuviera por debajo del 1\%. En este caso la variable crítica es la determinación del peso seco de la madera. Procedimiento similar se utilizó para el blanqueo, donde la blancura tiene un error asociado al proceso de $\pm 0,7$. Por lo tanto pulpas con diferencia por sobre 1,5\% ISO pueden considerarse distintas. Sólo las refinaciones en el molino Jokro para curvas de refino quedan exentas de este análisis. Sin embargo el grado de drenabilidad ( $\left.{ }^{\circ} \mathrm{SR}\right)$ se determinó siempre por triplicado. 


\section{RESULTADOS Y DISCUSIÓN}

\section{Rendimiento y blanqueabilidad}

El efecto combinado entre el tiempo de reacción a $170^{\circ} \mathrm{C}$ y la carga de $\mathrm{Na}_{2} \mathrm{SO}_{3}$ puede verse en la figura 1. Llama la atención que el rendimiento pulpable aumentó con el incremento de la carga de reactivos. Teóricamente una mayor carga de reactivos significa condiciones de pulpaje más extremas, lo que produciría una deslignificación más intensa y pérdida en el rendimiento. La extraña correlación entre el rendimiento y la carga de reactivos, en este caso, se explica por la drástica caída del $\mathrm{pH}$ de la solución durante la cocción. La solución de sulfito de sodio tiene un pH inicial cercano a 9,7, el cual desciende al entrar en contacto con la madera debido, principalmente, a las reacciones de deacetilación de xilanas y la neutralización de ácidos orgánicos presentes en la madera. La pérdida de grupos acetilos en madera de latifoliadas es de gran importancia, y puede llegar a significar hasta un $5 \%$ de pérdida en el rendimiento. La caída del $\mathrm{pH}$ debido a una insuficiente carga de sulfito (u otro agente buffer) genera condiciones apropiadas para que se inicien reacciones de hidrólisis de carbohidratos, lo cual genera una pérdida adicional de rendimiento, dañando de paso la selectividad del proceso. Rydholm (1965) señala que una caída en el valor del pH bajo 6 provoca una aceleración de éste proceso de hidrólisis, el cual se ve catalizado a altas temperaturas. Se estimó que para obtener un $\mathrm{pH}$ final sobre 6 es necesario una carga mínima de $\mathrm{Na}_{2} \mathrm{SO}_{3}$ de $16,5 \%$ base madera seca (bms), valor que coincide con el aumento en la velocidad de pérdida de rendimiento (Fig. 1. curva de $60 \mathrm{~min}$ ).

Otro efecto negativo que evidenció la disminución de la carga de $\mathrm{Na}_{2} \mathrm{SO}_{3}$ es la blancura de la pulpa post refinado (Fig. 2). Ésta disminuye drásticamente si la carga de $\mathrm{Na}_{2} \mathrm{SO}_{3}$ baja de $15 \%$ (bms). Estos experimentos confirman los obtenidos por Area et al. (2002) donde indican que el $\mathrm{Na}_{2} \mathrm{SO}_{3}$ tiene un efecto positivo sobre la blancura de la pulpa, no así el $\mathrm{Ca}_{2} \mathrm{CO}_{3}$. Se observa en la figura 2, que un aumento en la carga de sulfito más allá de $20 \%$, no aporta mayor aumento en la blancura. La blancura de la pulpa después de la etapa química es, para la obtención de pulpa semiquimíca blanqueda, determinante.

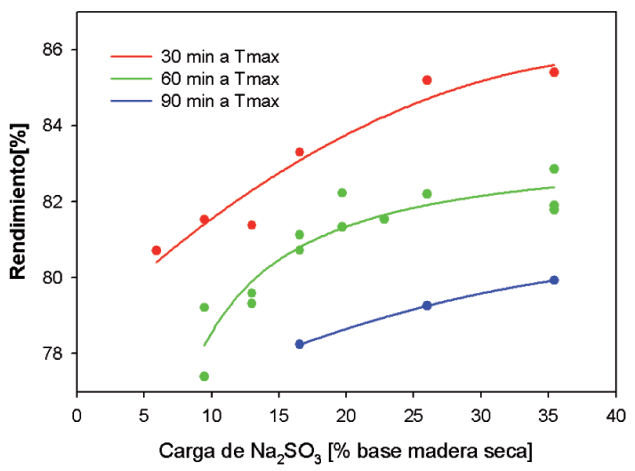

Fig. 1. Rendimiento pulpable v/s carga de sulfito de sodio. Tiempo de calentamiento $90 \mathrm{~min}$. Temperatura máxima (Tmax) $170^{\circ} \mathrm{C}$.

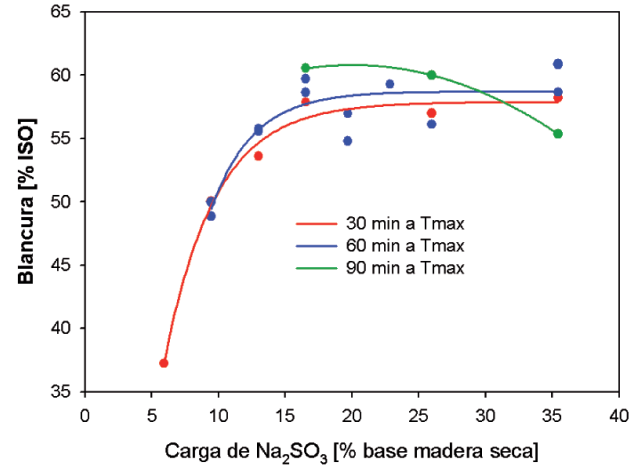

Fig. 2. Blancura v/s carga de sulfito de sodio. Tiempo de calentamiento $90 \mathrm{~min}$. Temperatura máxima (Tmax) $170^{\circ} \mathrm{C}$. 


\section{Grado de sulfonación y consumo de reactivos}

Dado la mayor complejidad en la recuperación de reactivos de los procesos al sulfito, la optimización de la carga de reactivo en el pulpado NCCS se torna un factor crítico. La figura 3, muestra el consumo de reactivos en función del rendimiento pulpable. Se puede destacar que a mayor carga de reactivo, mayor es el consumo específico de sulfito. Esto se explica, en parte, al mayor grado de sulfonación de la pulpa (Fig.4). Importante es destacar que para rendimientos superiores a $84 \%$ (tiempos cortos de reacción) el consumo de sulfito es, en el rango de carga entre 16.5 y $35.5 \%$ similar, lo que indicaría que la velocidad de reacción del sulfito con la madera es, en el inicio del proceso, poco dependiente de la concentración y se puede inferir que, a medida que el rendimiento baja (sobre los 30 min de cocción), la velocidad de reacción es más dependiente de la concentración.

El aumento en la carga de sulfito, en la etapa inicial de pulpaje, no necesariamente se refleja en un mayor consumo. El sulfito residual en el licor para cargas superiores a $20 \%$ bms es igual o superior al 50\% del inicial. Este resultado indica que es aconsejable la implementación de un sistema de recuperación basado en el reciclo del licor agotado (resultados no mostrados), el cual contiene, para muy altos rendimientos y carga de sulfito, aun una alta carga de sulfito activo residual y baja carga orgánica, disminuyendo así el make up de reactivo aplicado y mejorando la relación sólidos orgánicos/ sólidos inorgánicos del licor final.

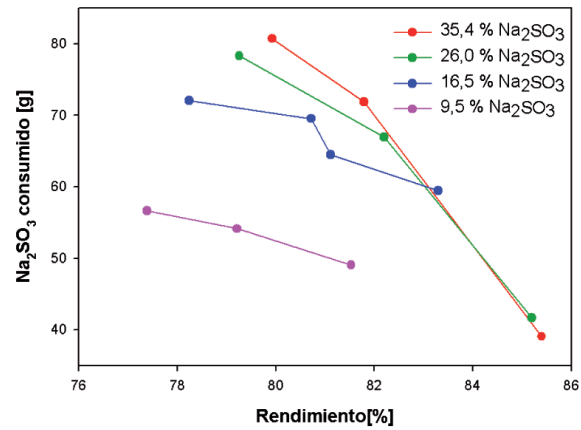

Fig. 3. Sulfito consumido en función del rendimiento pulpable. Tiempo de calentamiento $90 \mathrm{~min}$. Temperatura máxima $(\operatorname{Tmax}) 170^{\circ} \mathrm{C}$

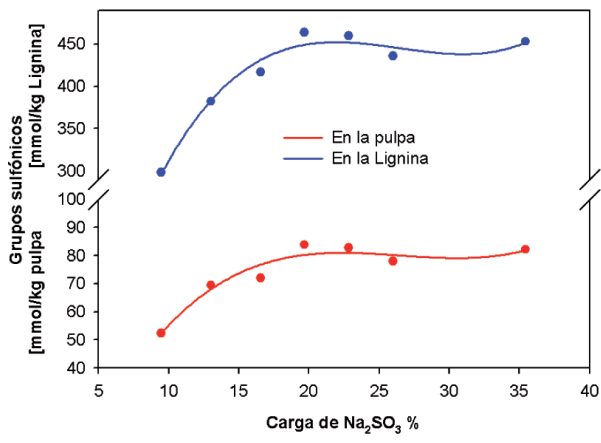

Fig. 4. Grado de sulfonación de la pulpa v/s carga de sulfito de sodio. Tiempo de calentamiento $90 \mathrm{~min}$. Tiempo a Tmax 60 min. Temperatura máxima (Tmax) $170^{\circ} \mathrm{C}$.

El grado de sulfonación de la pulpa (Fig. 4) se relaciona a menudo con las propiedades físicomecánicas de ésta y con la facilidad para la desfibración de las astillas (Heitner y Hattula 1988). En la figura 4 se observa, que un aumento en la carga de sulfito, más allá de $20 \%$ bms no tiene mayor efecto sobre el grado de sulfonación. Al realizar un balance de materia para el $\mathrm{Na}_{2} \mathrm{SO}_{3}$ se obtiene que, a un determinado rendimiento (ej. $82 \%$ ), el $\mathrm{Na}_{2} \mathrm{SO}_{3}$ consumido (65g) es mucho mayor que el reflejado en la pulpa como grado de sulfonación (12,5g). La explicación de dicho fenómeno encuentra dos posibilidades no excluyentes; El grado de sulfonación de la lignina disuelta es al menos 4 veces mayor que el de la lignina remanente y/o el consumo de sulfito, mediante reacciones secundarias, como por ejemplo oxidación de grupos terminales de carbohidratos, es muy alta (Sixta 2006, Rydholm 1965). 


\section{Consumo de energía y propiedades físico mecánicas.}

El consumo de energía, así como el rendimiento del proceso, se encuentran directamente relacionados con las propiedades físico-mecánicas de las pulpas semiquímicas. En los procesos al sulfito juega también un rol importante el grado de sulfonación. En la figura 5 se observa el grado de refino alcanzado para pulpas de igual rendimiento, pero obtenidas con cargas de reactivo variable. Se concluye que las pulpas producidas con mayor carga de sulfito son más fácilmente refinables, puesto que requieren de un menor tiempo de refino (menor energía) para alcanzar un determinado grado de refinación. Esto debido al mayor grado de sulfonación (Heitner y Hattula 1988) y la mejoría de selectividad del proceso con mayores dosificaciones de sulfito (Pereira 2009, Rehbein et al. 2010).

En la figura 6 se muestra la dependencia entre el grado de refinación y el tiempo de refinado para pulpas con diferente rendimiento (alcanzado con diferentes tiempos de cocción), obtenidas con la misma carga de reactivo. Es claro ver que, para alcanzar un mismo grado de refinación, independiente de la carga de reactivo, las pulpas de mayor rendimiento, debido a su menor grado de deslignificación, requieren un tiempo de refino más largo que aquellas de menor rendimiento. Esta diferencia, en todo caso, se ve disminuida cuando la carga inicial de sulfito aumenta (carga 26\% bms). Existen aquí dos probables explicaciones; a mayor carga de sulfito la selectividad del procesos aumenta, por tanto, el aumento de rendimiento se debe a una mayor fracción de hemicelulosas $\mathrm{y}$, por otra parte, la pasta con mayor carga de sulfito alcanza un mayor grado de sulfonación, lo que le confiere mayor hidrofilicidad. Ambos efectos favorecen al refinado de la pasta.

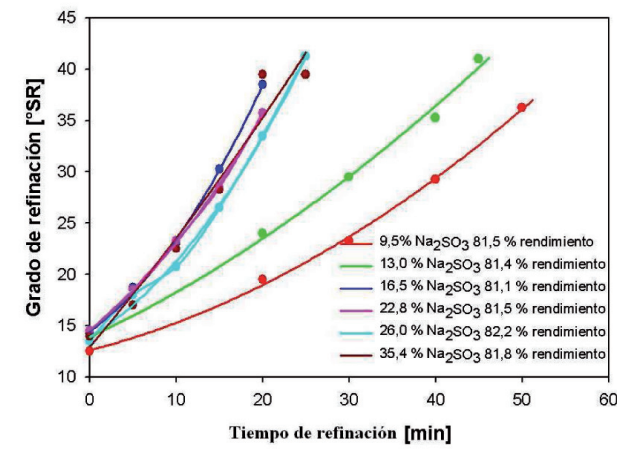

Fig. 5. Grado de refinación v/s tiempo de refinación. Rendimiento $81 \%$. Diferentes cargas de sulfito

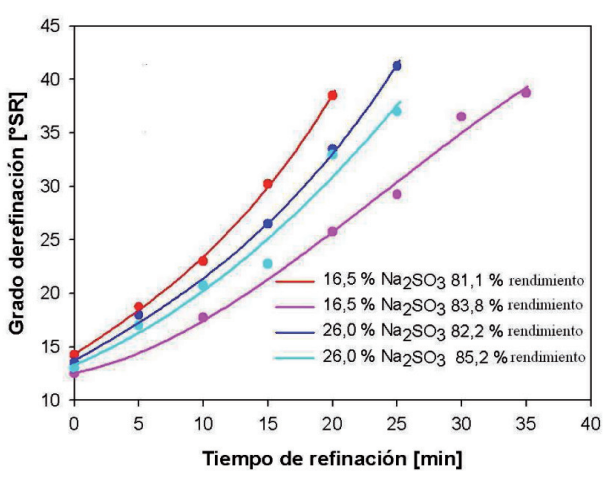

Fig. 6. Grado de refinación v/s tiempo de refinación. Rendimiento. Diferentes cargas de sulfito y rendimiento. Tiempo de cocción variable 30 y $60 \mathrm{~min}$

El efecto de la carga de $\mathrm{Na}_{2} \mathrm{SO}_{3}$ y la energía de refinación sobre el índice de tensión de las pulpas puede ser observado en la figura 7 . El rendimiento pulpable es para todas las muestra cercano a $81 \%$. Es importante notar que la mayoría de las pulpas, incluso aquellas de menor carga, presentan valores en su índice de tensión superiores a aquellas observadas en otros estudios (Area et al. 2004) (comparadas al mismo nivel de rendimiento). Se observa además, que el índice de tensión aumenta significativamente cuando la carga de sulfito de sodio aplicada en el pulpaje es superior a 13,0\%. Es importante notar también que el desarrollo de las propiedades físico-mecánicas de las pulpas con altas cargas de sulfito se logra con una menor energía consumida (menos tiempo de refinado, fig 6) y, a diferencia de lo normalmente visto para pulpa de alto rendimiento, los valores máximos de éstas son alcanzados a bajo grado de refinación (cerca de $30^{\circ} \mathrm{SR}$, en vez de 45 o más ${ }^{\circ} \mathrm{SR}$ ). 


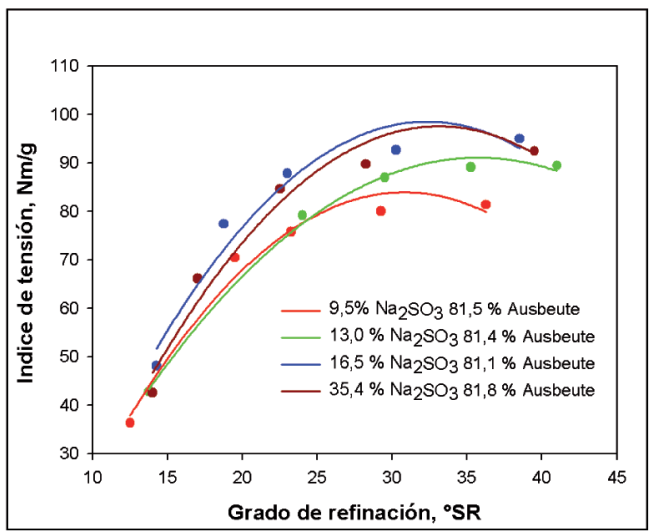

Figura 7. Índice de tensión v/s grado de refinación. Rendimiento 81\%.

Diferentes cargas de sulfito.

\section{Blanqueo de las pulpas.}

Para ver el efecto de la carga de peróxido sobre el blanqueo de la pulpa se escogió una pulpa con $82 \%$ de rendimiento una blancura inicial de $61,5 \%$. La blancura de la pulpa después del tratamiento con DTPA aumentó a $65,1 \%$ ISO. Esto debido a la remoción de metales que en combinación con estructuras cromofóricas intensifican el color.

En la figura 8 se observa el efecto de la carga de $\mathrm{H}_{2} \mathrm{O}_{2}$, temperatura y el tiempo sobre la blancura de la pulpa obtenida. Se destaca que, con cargas de $\mathrm{H}_{2} \mathrm{O}_{2}$ inferiores a $3 \%$ bps, se alcanzaron fácilmente valores en blancura superiores a $80 \%$ ISO. La mayor facilidad de blanquemiento de la pulpa semiquímica de $E$. globulus, con respecto a otras especies de eucaliptos, ha sido anteriormente observada por Area et al. (2004, 2002). Sin embargo en estos estudios tanto la carga como el consumo de reactivos de blanqueo fue significativamente mayor. El aumento de la temperatura y el tiempo, en el rango estudiado, tienen un efecto positivo sobre la blancura. Los mejores resultados se obtienen a $80^{\circ} \mathrm{C}$ y 180 min de reacción. Otro aspecto importante de destacar es el bajo consumo de peróxido, el cual se sitúa en todos los caso por debajo del 50\% (Fig. 9).

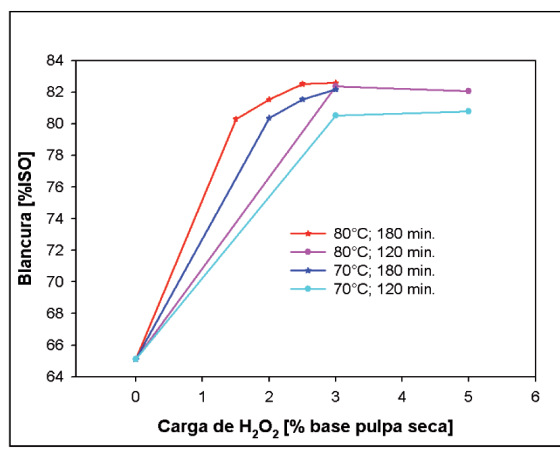

Fig. 8. Blancura v/s Carga de peróxido de hidrogeno. Pulpa 82\% Rendimiento. Quelación 01,\% DTPA.

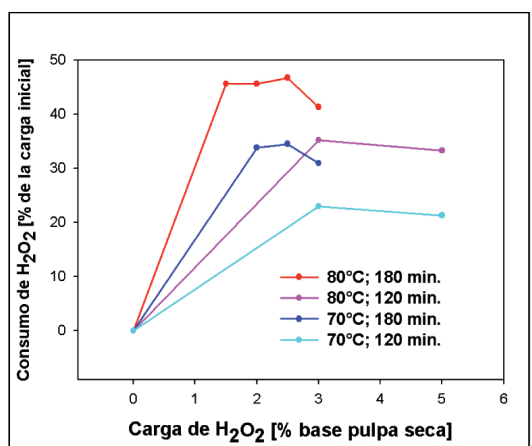

Fig. 9. Consumo de peróxido de hidrógeno durante el blanqueo de pulpa semiquímica

Las pulpas blanqueadas con $\mathrm{H}_{2} \mathrm{O}_{2}$ fueron finalmente sometidas a un blanqueo reductor con FAS. Los resultados se observan en la figura 10. El aumento de blancura, dependiendo de la blancura obtenida en la etapa de peróxido, oscila entre los 5 y 6 puntos para los casos usando $\mathrm{Na}_{2} \mathrm{SiO}_{3}$ como agente estabilizante del peróxido, lo cual es significativo dado el alto grado de blancura que la pulpa presentaba. Esta facilidad del blanqueo es atribuida en parte a la menor concentración de lignina residual en la superficie de las fibras (Pereira 2009, Rehbein et al. 2010) y, a la alta blancura inicial de las pulpas obtenidas mediante el pulpaje. 


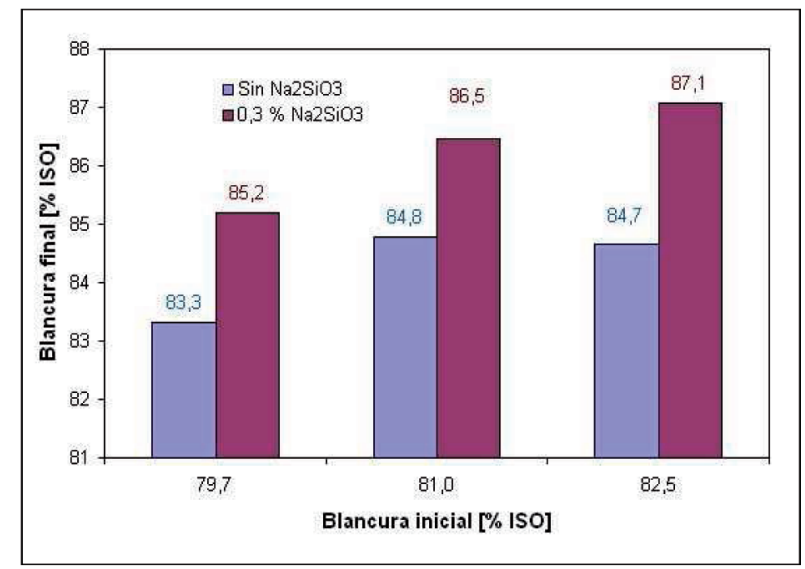

Figura 10. Etapa reductora con FAS de pulpa químico-mecánica preblanqueada con $\mathrm{H}_{2} \mathrm{O}_{2}$. Carga FAS: 0,5\% bps; T: $99^{\circ} \mathrm{C} ; \mathrm{pH}: 10,5$.

\section{CONCLUSIONES}

La utilización de una mayor dosis de sulfito de sodio durante el pulpaje NSSC de E. globulus tuvo como consecuencia un impacto positivo sobre las propiedades fisicomecánicas de la pulpa y su blanqueabilidad. Se concluye que una carga de sulfito por sobre $16 \%$ bps (sin el uso de otra fuente alcalina) es suficiente para mantener el $\mathrm{pH}$ a niveles adecuados que minimizan la pérdida de rendimiento pulpable debido a reacciones de hidrólisis ácida.

Bajo estas condiciones se obtienen pulpas con rendimientos superiores a $80 \%$ con propiedades físico-mecánicas comparables a una pulpa química (índice de tensión sobre $90 \mathrm{Nm} / \mathrm{g}$ ) con un grado de refino muy bajo $\left(30^{\circ} \mathrm{SR}\right)$, lo que se traduce en menores costos energéticos de refinación. La pulpa resultó ser también fácilmente blanqueable, alcanzando valores sobre $80 \%$ ISO con cargas de peróxido inferiores a $3 \%$ bps.

Por último un aumento de la dosis de sulfito, no se tradujo necesariamente en un mayor consumo de reactivo, permitiendo así reusar el sulfito residual mediante reciclo. 


\section{BIBLIOGRAFÍA}

Area, M.; Felissia, F.; Bengoechea, D.; Venica, A.; Valade, J. 2000a. Utilización de componentes orgánicos de licores residuales NSSC como aditivos en la fabricación de papeles y cartones. I Congreso Iberoamericano de Investigación en Celulosa y Papel.

Area, M, Sánchez, M.; Felissia, F.;Venica, A.; Valade, J. 2000b. Copolimerización graft de lignosulfonatos presentes en licores residuales del proceso NSSC. I Congreso Iberoamericano de Investigación en Celulosa y Papel.

Area, M.C.; Felissia F.; Venica A.; Valade J. 2001. NSSC process optimization: Pulping, pulps and spent liquors. Tappi Journal 84(4): 65-77.

Area, M.; Felissia, F.; Nuñez, C.; Venica, A. V. 2004. Estudio comparativo de especies de eucalyptus y su respuesta al pulpado NSSC; in: III concreso Iberoamericano de Investigación en celulosa y papel. Cordoba, España.

Area, M.; Felissia, F.; Clermont, J. E.; Venica, A. 2002. Optimización de la carga de $\mathrm{Na}_{2} \mathrm{SO}_{3}$ y $\mathrm{Na}_{2} \mathrm{CO}_{3}$ en el proceso NSSC de Eucalyptus Viminalis; in: II concreso Iberoamericano de Investigación en celulosa y papel. Campinas, Brasil.

Evtuguin, D.; Neto, P. 2007. Recent advances in eucalyptus wood chemistry: Structural features through the prism of technological response. In: $3^{\text {th }}$ International Colloquium on eucalyptus pulp. Belo Horizonte, Brasil.

Heitner, C.; Hattula, T. 1988. Ultra High yield Pulping VI. The Effect of Sulfonation On The Development Of Fiber Properties. J. Pulp Pap. Sci. 14(1): 6-11.

Ingruber, O. V. 1985. Pulp and Paper Manufacture: Vol. 4: Sulfite science and technology. The Joint Texbook Committee of the Paper Industry (TAPPI/CPPA). cap. VIII.

ISO Standards handbook. 1984. Pulps- ISO 5264/III-1979, Laboratory beating- Part III: Jokro mill Method. Paper, board and pulps. International Organization for Standardization, Switerland.

ISO Standards handbook. 1984. Pulps- ISO 5267/1-1979 Determination of drainability- Part 1: Schopper Riegler method. Paper, board and pulps. International Organization for Standardization, Switerland.

ISO Standards handbook. 1984. Pulps- ISO 5269/2-1980 Preparation of Laboratory sheets for physical testing- Part 2: Rapid Köthen method. Paper, board and pulps. International Organization for Standardization, Switerland.

Kordsachia, O. 2002. Stand und Perspektiven der Schwarzlaugenvergasung. IPW Science and Technology 10: 46-49.

Monsalve, E. 2010. Utilización de membranas de ultrafiltración para la recuperación de reactivos y materia orgánica en el proceso semiquímico al sulfito neutro. Memoria de titulo, Facultad de Ingeniería, Universidad de Concepción, Chile.

Pereira, M. 2009. Herstellung von Halb- und Vollzelstoffen aus Eucalyptus globulus Labill. Und Eucalyptus nitens Meiden aus Chile mit alkalischen Sulfitlösungen. Disertation, Universidad de Hamburgo, Alemania. 
Périn-Levasseur, Z. ; Maréchal, F. ; Paris, J. 2010. Analysis of a Biorefinery Integration in a Bisulfite Pulp Process. Pulp and Paper Canada. may/jun 2010. 31-33pp.

Rehbein, M.; Pereira, M.; Koch, M.; Kordsachia, O. 2010. Topochemical investigation into the delignification of Eucalyptus globulus chips during semi-chemical sulfite pulping. Wood Sci Technol 44: 435-449.

Rydhol, S.A. 1965. Pulping processes. Interscience Publications, New York.

SCAN Test Methods. 1988. Scandinavian Pulp, Paper and Board Committee, Sweden.

Sixta H. 2006. Handbook of pulp. Weiley-Vch Verlag GmbH \& Co KGaA, Weinheim.

TAPPI Test Methods. 1992. T410 om-93 Grammage of paper and paperboard (Weight per unit area). Standard Methods for Pulp and Paper, Technical Association of Pulp and Paper Industry, TAPPI Press, Atlanta.

TAPPI Test Methods. 1992. T404 om-92 Tensile breaking strength and elongation of paper and paperboard. Standard Methods for Pulp and Paper, Technical Association of Pulp and Paper Industry, TAPPI Press, Atlanta.

TAPPI Test Methods. 1992. T403 om-91 Bursting Strengh of Paper. Standard Methods for Pulp and Paper, Technical Association of Pulp and Paper Industry, TAPPI Press, Atlanta.

TAPPI Test Methods. 1992. T414 om-88 Internal tearing resistance of paper (Elmendorf-type method). Standard Methods for Pulp and Paper, Technical Association of Pulp and Paper Industry, TAPPI Press, Atlanta.

TAPPI Test Methods. 1992. T220 om- 88 Physical testing of pulp handsheets. Standard Methods for Pulp and Paper, Technical Association of Pulp and Paper Industry, TAPPI Press, Atlanta.

Xu, E. 2009. Chemical Mechanical Pulping Of Eucalyptus - Latest Development \& Comparison. In: $4^{\text {th }}$ International colloquium on Eucalyptus pulp. Concepción, Chile.

Zanuttini, M.; Citroni, M.; Martinez, MJ.; Marzocchi, V. 1998. Chemimechanical pulping of poplar wood. Alkaline wood pretreatment at low temperature. Holzforschung 52(4): 405-409.

Zanuttini, M.; Marzocchi, V.; Mocchiutti, P.; Inalbon, M. 2005. Deacetylation consequences in pulping processes. Holz als Roh- und Werkstoff 63(2): 149-153. 